\title{
Oxide nonlinear crystals: optical properties and phase-matching for terahertz wave generation
}

\author{
O. I. Potaturkin ${ }^{1}$, V. D. Antsygin ${ }^{1}$, A. A. Mamrashev ${ }^{1}$, N. A. Nikolaev ${ }^{1}$, \\ Yu. M. Andreev ${ }^{2,3}$, G. V. Lanskii ${ }^{2,3}$, V. A. Svetlichnyi ${ }^{3}$, K. A. Kokh ${ }^{4}$ \\ ${ }^{1}$ Institute of Automation and Electrometry SB RAS, Novosibirsk, Russia, potaturkin@iae.nsk.su \\ ${ }^{2}$ Institute of Monitoring of Climatic and Ecological Systems SB RAS, Tomsk, Russia \\ ${ }^{3}$ Siberian Physical Technical Institute of Tomsk State University, Tomsk, Russia \\ ${ }^{4}$ Institute of Geology and Mineralogy SB RAS, Novosibirsk, 630090, Russia
}

\section{Introduction}

Oxide nonlinear crystals such as beta-barium borate $\left(\beta-\mathrm{BaB}_{2} \mathrm{O}_{4}\right.$ or $\left.\beta-\mathrm{BBO}\right)$, potassium titanyl phosphate $\left(\mathrm{KTiOPO}_{4}\right.$ or KTP), potassium titanyl arsenate $\left(\mathrm{KTiOAsO}_{4}\right.$ or KTA) are widely used for efficient harmonic, difference frequency and optical parametric generation/oscillation in the visible and near-infrared ranges [1]. The crystals have wide transparency range stretching from $0.19 \mu \mathrm{m}$ for $\beta$-BBO, $0.35 \mu \mathrm{m}$ for KTP and KTA to near-IR or even mid-IR range. They also have moderate nonlinear coefficients and high optical damage threshold.

Recently these crystals attracted attention as prospective materials for terahertz applications. Several studies were devoted to measuring their optical properties (refractive index and absorption coefficient) in the terahertz domain [2-5]. Different schemes for IRto-THz and $\mathrm{THz}$-to- $\mathrm{THz}$ conversion were investigated [6-9]. Besides nonlinear crystals another attractive materials such as metals and semi-metals were considered for efficient conversion of near-IR femtosecond pulses to terahertz radiation on their surface [10].

In this study we measure terahertz optical properties and discuss possibilities of applying oxide nonlinear crystals, metals and semi-metals for terahertz wave generation.

\section{Selected results}

Absorption coefficient and refractive index dispersions in the $\mathrm{THz}$ range for all axes of nonlinear oxide crystals were measured at the room (RT), liquid nitrogen (LN), and liquid helium (LH) temperatures within the range of $0.2-2.5 \mathrm{THz}$ using custom-made terahertz time-domain spectrometer (THz-TDS) described elsewhere [2].

Absorption coefficient and refractive index of $\beta$ BBO crystals for ordinary and extraordinary waves were measured at RT and LN temperatures of $293 \mathrm{~K}$ and $81 \mathrm{~K}$, respectively. Sellmeier equations for both $o$ - and $e$-waves were formulated. Dispersion equations for at RT valid in the range of $187-10000 \mu \mathrm{m}$ :

$n_{o}^{2}=2.040+\frac{0.816 \lambda^{2}}{\lambda^{2}-12815}, n_{e}^{2}=2.478+\frac{0.160 \lambda^{2}}{\lambda^{2}-15598}$

Dispersion equations at LN temperature valid in the range of $182-3530 \mu \mathrm{m}$ for $o$ - and $144-2727 \mu \mathrm{m}$ for ewave:

$n_{o}^{2}=4.612+\frac{3.117 \lambda^{2}}{\lambda^{2}-16175}, n_{e}^{2}=5.970+\frac{0.800 \lambda^{2}}{\lambda^{2}-16659}(2)$
Using resulting dispersion curves we were able to find phase-matching (PM) for down-conversion or difference frequency generation of near-IR radiation into the $\mathrm{THz}$ range was found possible by $o-O \rightarrow e, e-$ $e \rightarrow o$ and original $e-e \rightarrow e$ types (Fig. 1) of three-wave interactions.

It was found that despite the significant decrease in the absorption coefficient at LN temperature, refractive index components and birefringence did note change a lot keeping PM conditions favorable for efficient down-conversion into the $\mathrm{THz}$ range.

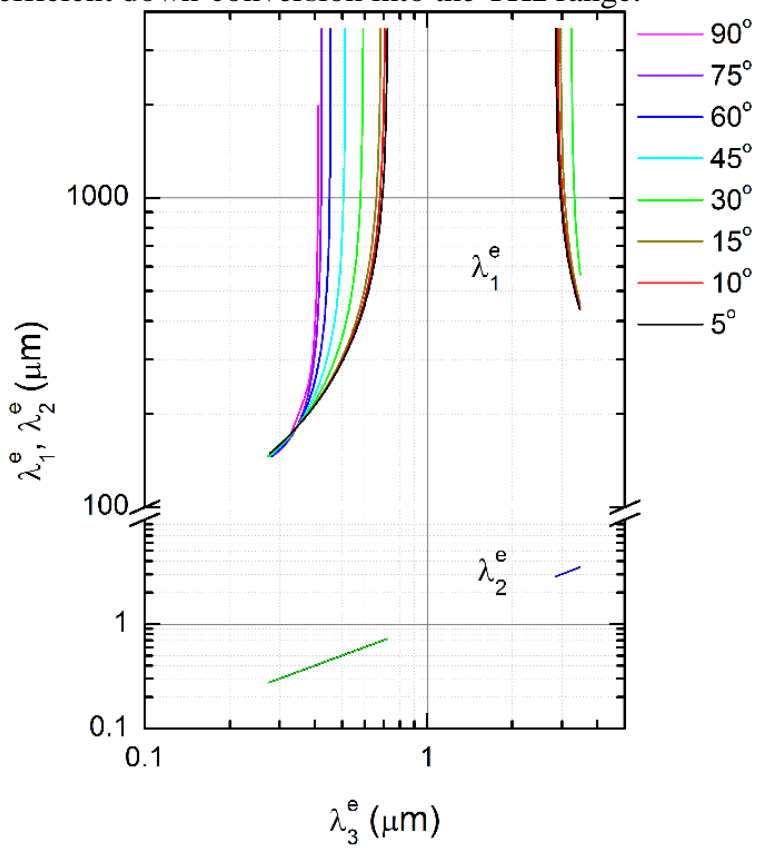

Fig. 1. Phase-matching curves for type $\mathrm{I}(e-e \rightarrow e)$ threewave interactions in $\beta$-BBO at RT. $\theta$ angles are shown in the figure inset

In a similar manner, terahertz optical properties of KTP crystals were measured at three temperatures. The refractive index for all three optical axes of KTP crystal was approximated in the form of Sellmeier equations. Here is a set of equations for LH temperature of $5.3 \mathrm{~K}$ where $\lambda$ is in $\mu \mathrm{m}$ :

$$
\begin{aligned}
& n_{x}^{2}=8.67715+\frac{1.34263 \lambda^{2}}{\lambda^{2}-11458} \\
& n_{y}^{2}=8.86222+\frac{1.22979 \lambda^{2}}{\lambda^{2}-12045} \\
& n_{z}^{2}=13.18625+\frac{0.86157 \lambda^{2}}{\lambda^{2}-21447}
\end{aligned}
$$


Using dispersion equations for the visible, infrared and terahertz ranges we obtained comprehensive results for phase-matched difference frequency generation in KTP crystals at different temperatures and different wavelengths presented in the Fig. 2.

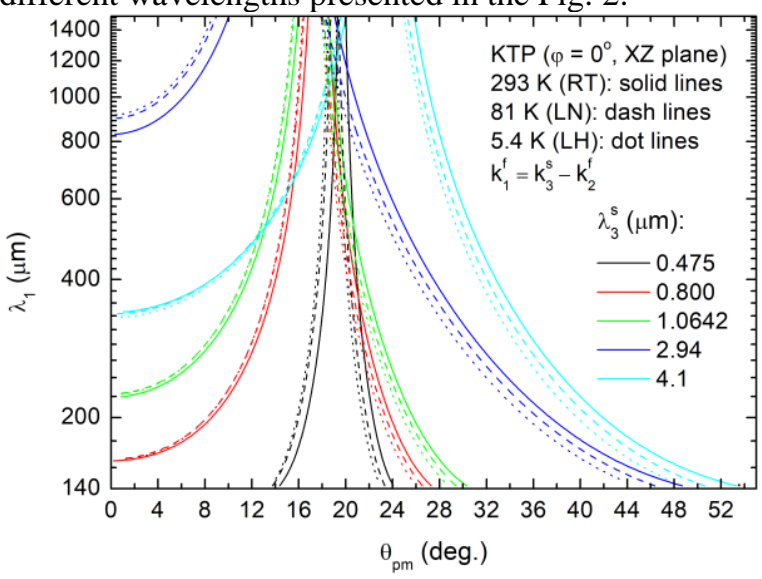

Fig. 2. Phase-matching curves for $s-f \rightarrow f$ type of difference frequency generation in the $\boldsymbol{X Z}$ principle plane of KTP crystal at RT (bold lines), LN (dash lines) and LH (dot lines) temperatures for different $\lambda$ from 0.475 to $4.1 \mu \mathrm{m}$

KTA refractive index measured at RT was approximated in the form of the following Sellmeier equations in the range from $132 \mu \mathrm{m}\left(n_{\mathrm{x}}\right), 155 \mu \mathrm{m}\left(n_{\mathrm{y}}\right)$, $313 \mu \mathrm{m}\left(n_{\mathrm{z}}\right)$ to $3200 \mu \mathrm{m}$ :

$$
\begin{aligned}
& n_{x}^{2}=9.70796+\frac{1.24017 \lambda^{2}}{\lambda^{2}-12935} \\
& n_{y}^{2}=9.62652+\frac{1.44084 \lambda^{2}}{\lambda^{2}-17674} \\
& n_{z}^{2}=16.6706+\frac{0.12511 \lambda^{2}}{\lambda^{2}-85811}
\end{aligned}
$$

Analogous phase-matching curves for DFG of Nd:YAG laser in KTA crystals is shown in the Fig. 3.

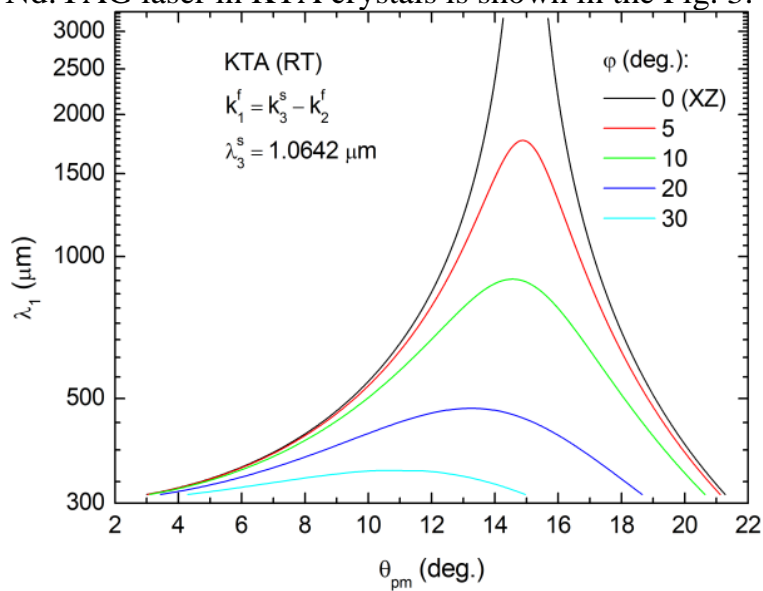

Fig. 3. Phase-matching curves for $s-f \rightarrow f$ type of difference frequency generation in KTP crystal at RT for the wavelength of Nd:YAG lasers

A common feature of all three abovementioned crystals was that cooling them down did not significantly affect phase-matching conditions. At the same time their absorption coefficient lowered drastically making cooled crystals more effective for terahertz wave generation.
The crystals were tested as narrowband $\mathrm{THz}$ radiation sources for a homemade spectrometer described elsewhere [11] having a long $(>1 \mathrm{~km})$ measuring path which consists of a transceiver adopted from a lidar complex [12].

We also studied terahertz generation on the surface of metals ( $\mathrm{Ti}, \mathrm{Ni}, \mathrm{Au}, \mathrm{Cu})$ and their alloys (Ti50.5Ni49.5) pumped by femtosecond Ti:Sapphire laser. The power of generated radiation was measured using Golay cell. Fig. 4 shows that the output signal is linearly depends on the pump pulse energy. We also found that the efficiency of optical-to-terahertz conversion is proportional to the material conductivity.

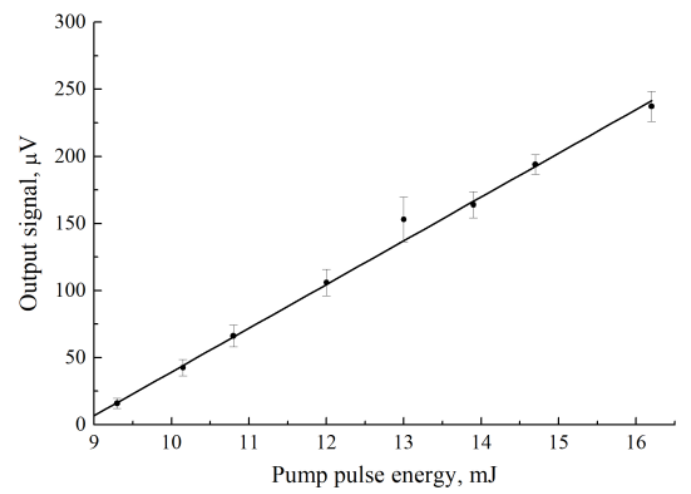

Fig. 4. Dependence of Golay cell signal proportional to the terahertz power generated on the surface of $\mathrm{Ti}_{50.5} \mathrm{Ni}_{49.5}$ alloy on the pump pulse energy

\section{References}

1. Nikogosyan D. Nonlinear optical crystals: a complete survey. New York: Springer, 2005.

2. Antsygin V.D. et al. Optical properties of borate crystals in terahertz region // Optics Communications. 2013. Vol. 309. P. 333-337.

3. Antsygin V.D. et al. Terahertz optical properties of potassium titanyl phosphate crystals // Optics Express. 2014. Vol. 22, Is. 21. P. 25436.

4. Andreev Y.M. et al. Observation of a different birefringence order at optical and $\mathrm{THz}$ frequencies in LBO crystal // Optical Materials. 2017. Vol. 66. P. 94-97.

5. Nikolaev N.A. et al. Terahertz optical properties of LBO crystal upon cooling to liquid nitrogen temperature // Quantum Electronics. 2018. Vol. 48, Is. 1. P. 19-21.

6. Huang J.-G. et al. Phase matching in RT KTP crystal for down-conversion into the $\mathrm{THz}$ range // Laser Phys. Lett. 2018. Vol. 15, Is. 7. P. 075401.

7. Mamrashev A.A. et al. Optical Properties of KTP Crystals and Their Potential for Terahertz Generation // Crystals. 2018. Vol. 8, Is. 8. P. 310.

8. Nikolaev N.A. et al. Optical properties of $\beta$-BBO and potential for $\mathrm{THz}$ applications // Journal of Physics: Conference Series. 2018. Vol. 951. P. 012003.

9. Nikolaev N.A. et al. Temperature dependence of terahertz optical properties of LBO and perspectives of applications in down-converters // Journal of Physics: Conference Series. 2018. Vol. 951. P. 012005.

10. Mironov V.A., Oladyshkin I.V., Fadeev D.A. Optical-to- $\mathrm{THz}$ radiation conversion on a semi-metal surface // Quantum Electronics. 2016. Vol. 46, Is. 8. P. 753-758

11. Huang J.-G. et al. Remote imaging by nanosecond terahertz spectrometer with standoff detector // Russian Physics Journal. 2018. Vol. 60, Is. 9. P 1638-1643.

12. Andreev Yu. M., Geiko P.P., Sherstov I.V. Development and testing of the lidar gas analyzing complex // Proc. SPIE. 1999. Vol. 3983, P. 386-394. 